\title{
Laboratory Diagnostics Performance in Uganda: A Survey of Test Availability and Constraints Across 100 Laboratories
}

Noel Namuhani ( $\nabla$ noelnamuhani@gmail.com )

Makerere University

Suzanne N Kiwanuka

Makerere University

Martha Akulume

Makerere University

Simeon Kalyesubula

Makerere University

William Bazeyo

Makerere University

Angela N. Kisakye

Makerere University

\section{Research Article}

Keywords: Functionality, test menus, supplies, Laboratories, Hubs, Uganda

Posted Date: September 23rd, 2021

DOl: https://doi.org/10.21203/rs.3.rs-842323/v1

License: (9) (i) This work is licensed under a Creative Commons Attribution 4.0 International License. Read Full License 


\section{Abstract \\ Background}

Clinical laboratory services are a critical component of the health system for effective disease diagnosis, treatment, control and prevention. However, many laboratories in Sub Saharan Africa remain dysfunctional. The high costs of tests in the private sector also remain a hindrance to accessing testing services. This study aimed at assessing the functionality of laboratories based on test menus and the associated constraints in Uganda.

\section{Methods}

This cross sectional quantitative study involved an assessment of 100 laboratories randomly selected in 20 districts from four regions of the country. Sixteen percent of the studied laboratories were regional hub laboratories. Laboratory in charges and managers in each of the selected laboratories were interviewed. A checklist for laboratory supplies adapted from the Essential Medicines and Health supplies list for Uganda, (2012) was used to assess availability of testing supplies. Data was analyzed using excel and STATA 14.

\section{Results}

At the point of assessment, generally, all laboratories were able to perform malaria tests and HIV tests. All the hub laboratories conducted malaria tests and TB screening. Less than half had electrolytes tests due to lack of equipment, nonfunctioning equipment and lack of reagents. Full blood count tests were missing in $25 \%$ of the hub laboratories mainly due to lack of equipment. The lack of reagents $(66.7 \%)$ and the lack of equipment (58.3\%) caused the majority $10 / 16$ of the hubs to routinely referred specimens for tests that are supposed to be carried out in these laboratories due to lack of reagents $(66.7 \%)$ and non-functional equipment (58.3\%). Although officially recognized as an operational structure, Hub laboratories lacked a list of essential and vital supplies.

\section{Conclusions}

Most laboratories performed well for the common tests. However, many laboratories did not meet testing requirements especially for the advanced tests according to standard testing menus for Uganda due to non-functioning equipment, lack of equipment and reagents. Hubs lack list of essential supplies. Therefore, there is need to provide equipment to laboratories, repair the non-functional ones and develop an essential list of supplies for the hub laboratories.

\section{Background}


Clinical laboratory services are a critical component of the health system because they aid effective disease diagnosis, treatment, control and prevention (1). Over the past decade, there has been an increased demand for laboratory testing services to manage infectious and non-infectious diseases including emerging and re-emerging diseases (1).

In Uganda, laboratory services are a key driver for effective delivery of the Uganda National Minimum Health Care Package (UNMHCP) (2). However, most of the laboratories in Uganda function below capacity and are characterized by un availability of vital tests, tests of low quality and clinically unreliable results (3). The quality of laboratory services has been hindered by a number of factors including lack of standardized laboratory test menus, equipment, techniques, supplies list for the various levels of healthcare delivery and among others $(4,5)$. In order for laboratories to operate efficiently and costeffectively, they need uninterrupted supply of reagents, functioning equipment, supplies and personnel. The inability to conduct tests delays and disrupts clinical care, prevention activities and public health programs (6).

A number of initiatives have been implemented to improve the quality of testing service in African laboratories $(7,8)$. Some of these include the establishment of the East Africa Public Health Laboratory Network Project, World Health Organization (WHO) which is reported to have led to the establishment of initiatives like Stepwise Laboratory Quality Improvement Process Towards Accreditation (SLIPTA), Strengthening Laboratory Management Toward Accreditation (SLMTA) (9). In 2013, Uganda embarked on the process of standardizing the laboratory test menus and supplies for the various tiers of laboratories in the laboratory network with the aim of improving quality of testing services (10). Despite these advances most of the laboratories in the country are still not performing to the set standards. Therefore, we established the functionality of the laboratories based on availability of test menus and supplies across the different laboratory levels in Uganda.

Laboratory service testing menus in Uganda: Laboratory testing menus are intended to define the minimum testing services expected at the various levels in the tiered laboratory network (10). Uganda has a well-defined laboratory tiered network that is aligned with the public health service delivery. At the lowest level, closest to the community, Health centre two ( $\mathrm{HCII}$ ) the basic laboratory tests are offered and subsequently at each higher levels of service delivery those tests which are not available at the lower levels are offered.

Laboratory Hubs: In order to increase access to quality laboratory services and improve accuracy, efficiency and immediacy in disease diagnosis, laboratory hubs were established in Uganda in 2006. Laboratory hubs provide enhanced services on-site or refer to a higher-level facility that has capacity to perform the test (11). The laboratory test menus for laboratory at each level of service delivery are summarized in Figure 1.

The functionality of the laboratories based on test menus and availability of supplies remains unknown in Uganda (3). This information deficit is a detriment to public health because it undermines service delivery and quality improvement efforts. This study therefore sought to assess laboratory 
functionality based on test menus and supplies availability in Uganda. This study presents policy makers and public health authorities in Uganda, with viable information for strengthening laboratory testing services.

\section{Methods}

\section{Study design and setting}

This cross-sectional study was conducted in the four regions (Northern, Western, West Nile, and Eastern) of Uganda.

\section{Study population and sampling}

A total of 100 laboratory managers and in-charges from 100 randomly selected laboratories participated in the study. This sample of 100 laboratories, considered to be representative of the four regions of the country was obtained by including five laboratories selected from each of the five districts randomly selected from each region. These included $57 \mathrm{HC}$ IIIs, $18 \mathrm{HC}$ IVs, 19 Hospitals and 6 regional referral hospital laboratories. Of all the 100 laboratories assessed, 16 were hub laboratories while 84 were non hub laboratories.

\section{Data collection procedures}

The data was collected between December 2015 and January 2016. Data on availability of testing services, and diagnostic capacities were assessed using a laboratory assessment tool adapted from the WHO and USAID assessment tool for laboratory services and supply chain (ATLAS), 2006. A checklist for laboratory supplies adapted from the Essential Medicines and Health supplies list for Uganda, (2012) was used to assess availability of testing supplies. Survey questions were administered in person to the laboratory managers or in charges at each laboratory facility. Test menus, specimen and test referrals, availability of supplies and reasons for lack of tests were documented at each laboratory level.

\section{Data management and analysis}

Quantitative data were entered in EPI Info version 7 software, cleaned and analyzed using Excel and STATA. Test and supplies availability were obtained by comparing the available test and supplies with standard testing menus (WHO, 2017) and standard essential lists for supplies respectively based on the level of health facilities (WHO, 2012). Proportions of facilities offering a given tests based on the standard testing menus and availability of supplies were obtained.

\section{Results}

In this section, we present the tests performed at the different laboratory levels based on the standard menus, reasons for the lack of tests, specimen and test referral and availability of vital and essential supplies. 


\section{Background characteristics}

A total of $57 \mathrm{HCllls}, 18 \mathrm{HCVs}, 19$ hospital laboratories and 6 regional referral hospital laboratories were assessed. Sixteen of these laboratories were hubs and 84 were non hubs. The laboratories had been in operation for an average of 15 years (Table 2).

Table 1: Levels of Laboratories assessed

\begin{tabular}{ll} 
Level of Laboratory & Frequency $(\mathbf{N}=\mathbf{1 0 0})$ \\
\hline HC III & 57 \\
\hline HC IV & 18 \\
\hline Hospital & 19 \\
\hline Regional Referral & 6 \\
\hline Hub laboratories & 16 \\
\hline Non Hub laboratories & 84
\end{tabular}

\section{Tests performed at facility level according to Test menus}

Health center III laboratories: Most health center III laboratories can conduct basic urine/stool analysis with a few exceptions due to non-functioning equipment and lack of reagents. All conducted HIV tests, malaria and pregnancy tests. None of the HC III laboratories performed blood grouping, sickle cell screening due to non-functioning equipment, lack of equipment and reagents. Full blood count and basic chemistry tests were grossly lacking due to non-functioning equipment, lack of equipment, reagents and skilled staff (Table 3).

Health center IV laboratories: All the Health center IV laboratories conducted HIV tests, malaria and pregnancy tests. None of the pneumonia and ulcer tests, electrolytes and chemistry tests was performed at Health center IV laboratories due to non-functioning equipment, lack of equipment, reagents and limited staff skills. The majority of the diabetes (83.3\%), Hepatitis (76.5\%) and CBC (60.0\%) tests were referred mainly due to broken down equipment and lack of reagents (Table 3 ).

Table 2: Tests performed at Health Center IIIs and Health center IV according to test menus 
Test Menus

Bacteriology tests

Stool analysis

Urine Analysis

AFB (TB screening)

Serology

Syphilis test

HIV test

Pregnancy test

Rapid Blood Sugar

Parasitology

Malaria test

Hematology

Full blood count

Blood grouping

Sickle cell screening test

Chemistry test

Test Menus for HCIV

Bacteriology

Stool analysis

Urine Analysis

AFB (TB screening)

Serology

Syphilis test

HIV test

Pregnancy test

Rapid Blood Sugar

Parasitology

Malaria test
HC III 57(\%) Reasons for unavailability of tests

$\begin{array}{ll}36(63.2) & * \& \\ 48(84.2) & * \& \\ 40(70.2) & * \# \&\end{array}$

52 (91.2) *\#\&

$57(100.0)$

$57(100.0)$

44(77.2) \&

$57(100.0)$

$-$

5 (8.8) *\#\&

O(0.0) *\#\&

$0(0.0) \quad$ *\#\&

5 (8.8) $\quad$ \$*\#\&

\section{HCIV 18 (\%)}

$16(88.9) \quad \&$

17 (94.4) \#

$17(94.4) \quad \&$

16 (88.9) *\#

18(100.0)

18(100.0)

3(16.7) \&


Electrolytes $\quad 0(0.0) \quad \$ * \# \&$

Hematology

\begin{tabular}{lll}
\hline Full blood count & $6(33.3)$ & *\#\& \\
\hline Blood grouping & $9(50.0)$ & $\&$ \\
\hline Sickle cell screening test & $7(38.9)$ & $\&$ \\
\hline Chemistry test & $1(5.6)$ & $\$ \star \# \&$
\end{tabular}

$\$$-Staff not trained, *-nonfunctioning equipment, \#-No equipment, \&-No reagents, bold-weak areas

\section{Tests performed at Hospital laboratories}

Almost all tests supposed to be conducted at hospital level laboratories, were conducted except for chemistry and electrolytes tests. Majority (52.6\%) of laboratories did not conduct electrolytes tests due nonfunctioning equipment, lack of equipment, reagents and skilled staffs. A few laboratories did not conduct chemistry tests $(26.3 \%)$ due to nonfunctioning equipment and lack of equipment. BAT tests (12.8\%), TB tests (16.7\%) and Ulcer tests (25.0) were also not available at the time of assessment due to lack of reagents and equipment (Table 4).

\section{Tests performed at Regional Referral hospital laboratories}

Almost all tests supposed to be conducted at regional referral laboratories were available at the time of the assessment. However, half of the laboratories did not conduct electrolyte tests due to lack of reagents and equipment. One lab did not conduct full blood count due to lack of reagents (Table 4).

Table 3: Tests Performed at Hospital and Regional Referral hospital laboratories according to testing menus 
Test Menus

Bacteriology

Stool analysis

Urine Analysis

AFB (TB screening)

Serology

Syphilis test

HIV test

Pregnancy test

Rapid Blood Sugar

Parasitology

Malaria test

Electrolytes

Hematology

Full blood count

Blood grouping

Sickle cell screening test

Chemistry test

Test Menus

\section{Bacteriology}

Stool analysis

Urine Analysis

AFB (TB screening)

Serology

Syphilis test

HIV test

Pregnancy test

Rapid Blood Sugar

Parasitology
Hospital 19(\%) Reasons for unavailability of Tests

$19(100.0)$

$19(100.0)$

$19(100.0)$
18 (94.7) \#

$19(100.0)$

$19(100.0)$

19 (100.0)
$19(100.0)$

9 (47.4)

16 (84.2)

18 (94.7)

18 (94.7)

14 (73.7)

RRH 6(\%)
*\#

\&

$\&$

*\#

Reasons for unavailability of Tests
(

*\#\$\& 
Malaria test

Electrolytes

Hematology

Full blood count

Blood grouping

Sickle cell screening test

Chemistry test
$6(100.0)$

$3(50.0)$

\#\&

\&

$6(100.0)$

$6(100.0)$

6(100.0)

\$-Staff not trained, *-nonfunctioning equipment, \#-No equipment, \&-No reagents, bold-weak areas

Tests performed at hub laboratories according to test menus and reasons for non-availability of tests

Almost all tests could be performed at hub laboratories. All the hub laboratories had malaria tests and TB screening. Less than half(43.8\%) of the hub laboratories were able to perform electrolytes tests due to lack of equipment, non-functioning equipment and lack of reagents, $1 / 4$ of the hub laboratories could not conduct Full blood count due to lack of equipment. A few 3/16 hub laboratories were not able to conduct chemistry tests due to lack of equipment and nonfunctioning equipment (Table 5).

Table 4: Tests available at Hub laboratories according to Test menus

\begin{tabular}{lll} 
Test Menus & N16 (\%) & Reasons for unavailability of tests \\
\hline Bacteriology & & \\
\hline Stool analysis & $14(87.5)$ & $\&$ \\
\hline Urine analysis & $14(87.5)$ & $\# \&$ \\
\hline AFB (TB screening) & $16(100.0)$ & - \\
\hline Serology & & \\
\hline Syphilis test & $14(87.5)$ & $\#$ \\
\hline Parasitology & & \\
\hline Malaria test & $16(100.0)$ & - \\
\hline Electrolytes & $\mathbf{7 ( 4 3 . 8 )}$ & *\#\& \\
\hline Hematology & & \\
\hline Full blood count & $\mathbf{1 2 ( 7 5 . 0 )}$ & $\#$ \\
\hline Chemistry & $\mathbf{1 3 ( 8 1 . 2 )}$ & *\#
\end{tabular}

\$-Staff not trained, *-nonfunctioning equipment, \#-No equipment, \&-No reagents, bold-weak areas 


\section{Tests and specimen routinely referred}

Generally, $48.4 \%$ of the laboratories referred specimen for tests that could be performed in the laboratories. This was mainly due to lack of reagents (44.1\%) and lack of equipment (39.8\%). More than half of laboratories at all levels received referred results more than a week from the time the specimens were referred, with HCIVs having the highest number $(68.8 \%)$ of laboratories receiving results after a week.

Health center III laboratories: Slightly less than half (42.1\%) of the health center III laboratories routinely referred tests for which they are mandated to perform. These included BAT Test (33.3\%), diabetes tests $(25.0 \%)$. The main reason for routine referral of these tests was lack of reagents.

Health center IV laboratories: Half 9/18 (50.0\%) of the health center IV laboratories routinely referred specimen for tests that could be carried out in the laboratory. These mainly included TB culture (9/9), CD4 (7/9), Viral load (9/13), chemistry (4/9) and hematology (3/9). The main reasons for referral included lack of reagents (88.8\%) and broken equipment (55.6\%).

Hospital level laboratories: More than half 11/19 (57.9\%) of the general hospital laboratories routinely referred specimen for tests that could be carried out in the laboratory. These mainly included PCR (11/11), Histology (7/11), TB culture (8/11) and Viral load (8/11). The main reasons for referral of tests included lack of reagents (72.7\%), broken equipment (72.7\%) and lack of staff $(9.1 \%)$.

Regional referral hospital laboratories: Less than half $(2 / 6)$ of the regional referral hospital laboratories routinely referred specimen for tests that could be carried out in the laboratory. These were mainly PCR, TB culture and viral load. The main reason for referral of specimens was lack of reagents.

Hub Laboratories: The majority $10 / 16$ of the hubs routinely referred specimens for tests that are supposed to be carried out in their laboratories. These tests included PCR (9/10), Histology (4/10), TB culture (10/10), chemistry (4/10), CD4 (6/10) and Viral load (10/10). The main reason for specimen referral in the hub laboratories included lack of reagents (66.7\%) and nonfunctional equipment (58.3\%).

\section{Availability of essential and vital laboratory supplies at different laboratory levels}

Overall: HIV testing kits, Malaria rapid tests strips, urine strips, were well stocked at all laboratory levels with over $95 \%$ of the laboratories having those supplies. At all levels, there was a general shortage of Igm (tubex), reconstituted laboratory reagents for malaria $\mathrm{CBC}$, TB and opportunistic infections which were found in less than half of the laboratories.

Health center III laboratories: Of the supplies mandated to be available at this level, majority (94.7\%) of the HC III laboratories were stocked with HIV testing kits (94.7\%), malaria rapid diagnostic tests (92.9\%) and about half (50.9\%) were stocked with blood glucose test strips. There were critical shortages in the availability of IgM tubex (8.8\%), reconstituted laboratory reagents for malaria, $\mathrm{CBC}$, tuberculosis and 
opportunistic infections (28.5\%), chemicals (25.2\%), blood grouping anti sera (42.8\%) and about $39.8 \%$ had glass ware and apparatus (Table 6).

Health center IV laboratories: For the supplies supposed to be available at Health center IV laboratories, all of them had HIV testing kits and malaria rapid test strips. None of the laboratories had CD4 Reagents, EasyCD 4 (GUAVA), Sysmex 3-part reagent kit, Cobas C 111 equipment reagents, Cobas c 311 liver profile test kits, reagents for Selectra, and blood collection sets and only $35.3 \%$ had been stocked with glassware (Table 7).

Hospital level laboratories: All hospital laboratories were stocked with HIV, malaria and urine test strips. There were critical shortages of CD4 reagents (20.0\%), Anti-microbial sensitivity disc kit (20.0\%), culture media $(16.0 \%)$, and coagulation reagents $(15.0 \%)$ Less than half of the laboratories were stocked with human liver function test kits (48\%), Blood grouping anti sera (48\%) and blood collection supplies $(44.0 \%)$ while none of them were stocked with reagents for selectra and biochemistry reflotron reagents (Table 6).

Hub level laboratories: Availability of supplies according to supplies menus at Hub laboratories level could not be assessed due to lack of a supplies list for these hub laboratories.

Table 5: Availability of selected Essential and Vital Supplies according to the menus at the time of assessment 


\begin{tabular}{|c|c|c|c|}
\hline Supplies Menus & $\mathrm{HC}$ III & HC IV & Hospitals $\mathrm{N}=\mathbf{2 5}$ \\
\hline HIV testing kits & $54(94.7)$ & $17(100.0)$ & $25(100.0)$ \\
\hline Specimen containers & 42(73.7) & $13(76.5)$ & $20(79.4)$ \\
\hline Blood glucose test strips & $29(50.9)$ & 7(41.2) & $21(84.0)$ \\
\hline Brucella abortus Ag & * & $5(29.4)$ & $14(56.0)$ \\
\hline HCG pregnancy test strips & $45(79.0)$ & 12(70.6) & $24(86.0)$ \\
\hline lgM (tubex) & $5(8.8)$ & $1(5.9)$ & $1(4.0)$ \\
\hline Malaria rapid & $53(92.9)$ & $17(100.0)$ & $25(100.0)$ \\
\hline RPR test strips & $48(84.2)$ & $13(76.5)$ & 19(76.0) \\
\hline Treponema & 15(26.3) & $6(35.3)$ & $15(60.0)$ \\
\hline Urine test strips & $51(91.1)$ & 16(94.11) & $25(100.0)$ \\
\hline Reconstituted lab reagents for malaria, CBC, TB & $16(28.5)$ & $7(41.2)$ & $11(43.3)$ \\
\hline Tuberculosis reagents kit & $48(84.2)$ & 15(88.2) & $25(100.0)$ \\
\hline Malaria fields stain reagents kit & $29(48.9)$ & $7(41.2)$ & $15(60.0)$ \\
\hline Gram stain kit & $32(55.7)$ & 12(70.6) & $24(95.0)$ \\
\hline Chemicals & $18(25.2)$ & $5(29.4)$ & $16(62.2)$ \\
\hline CD4 Reagents & * & $0(0.0)$ & $5(20.0)$ \\
\hline BD FACS Count reagents & * & $1(5.9)$ & $10(40)$ \\
\hline CD4 point of care machine & * & 12(70.6) & $9(36.0)$ \\
\hline PointCare Now & * & 2(11.8) & $1(4.0)$ \\
\hline HUMAN liver function test & * & $1(5.9)$ & $13(52.0)$ \\
\hline Blood grouping anti sera & $24(42.8)$ & 12(70.6) & $16(64.0)$ \\
\hline Blood collection sets & * & $0(0.0)$ & $7(28.0)$ \\
\hline Blood giving sets & * & $5(29.4)$ & $20(80.0)$ \\
\hline Anti-microbial sensitivity disc kit & * & * & $5(20.0)$ \\
\hline
\end{tabular}

* Supplies not in test menus for that level of health facility

\section{Discussion}


The functionality of the laboratories in terms of test menus is critical in guiding clinicians in diagnosing and giving the appropriate course of treatment to patients and also guiding public health workers on the effectiveness of health intervention and health programs (12). Therefore, the main purpose of this study was to establish the functionality of the laboratories based on availability of test menus and supplies across the different laboratory levels in Uganda. In this study, we found that many laboratories performed well regarding the common tests of malaria, HIV, urine and stool analysis, with low performance of advanced tests like chemistry, electrolytes and full blood counts in laboratories mandated to perform such tests. The main reasons for unavailability of tests was non-functioning equipment, lack of equipment and reagents.

The study indicated that generally, most basic tests were available at the time of assessment across the different laboratory levels. The most frequently done tests included malaria test, stool and urine analysis, Syphilis and TB screening. This finding concurs with the results of a survey in Kampala which revealed that stool analysis, urinalysis, syphilis and malaria tests were among the high availability tests in the city (13). However, many laboratories did not meet testing requirements especially for the advanced test according to standard testing menus for Uganda. A few HC III laboratories performed advanced tests which were not in their mandate. These could have been privately owned laboratories with the capacity to perform such tests.

It is indicated in this study that there was a dismal performance on the full blood count, Electrolytes and Chemistry tests at HC IV, hospital and regional referral levels where these tests are actually supposed to be conducted. The poor performance in these tests was highest at HCIV, which is the first point of referral. This dismal performance is attributed to mainly the lack of functional equipment for these tests. This underscores the need for regular maintenance of the equipment. Similar observations were reported in south western Ethiopia where clinical chemistry performance was very low and the quality of clinical chemistry test services were under standard (14). The most common reason for not having these tests was the lack of equipment followed by lack of reagents to run the tests. This is consistent with the findings of a study by (15) in Kampala, Uganda which revealed that $92 \%$ of the laboratories in reported that tests were not carried out due to lack of equipment and reagents. Thus, to improve the quality and availability of clinical chemistry, electrolytes and full blood count tests, there is need for measures to boost laboratories with essential and vital supplies and the key equipment.

Regarding functionality of hub laboratories, Although hub laboratories were equipped to perform full blood count, chemistry tests and electrolytes tests to service a wider area and act as referral lab for the region, $1 / 4$ of the hub laboratories could not perform FBC due to lack of equipment. Only less than half were able to conduct electrolytes due to non-functioning equipment, lack of equipment and reagents. Since these hub laboratories are intended to bridge gaps in service availability more, therefore efforts need to be made to ensure that the ability of hubs to conduct electrolyte and chemistry tests is not constrained by are equipped with chemistry equipment, repair non-functioning equipment and the lack of ensure reagents are available. 
The study revealed that about half of the laboratories routinely referred specimen for tests that they should be performing according to standard menus. These gaps were more pronounced in HCIV and hospitals laboratories experienced in over $56.0 \%$ of the laboratories. Furthermore, unexpectedly, a high number of hub laboratories routinely referred specimen for tests that they should be conducting compared to non-hub laboratories. The main reasons for referral of specimen was lack of reagents and functional equipment. According to the National laboratory strategic plan (2010-2015), lack of or frequent break down of equipment and frequent stock out of supplies were the reasons many laboratories did not fulfill the testing requirement. Hub laboratories offer a prime opportunity to further improve access and quality of services. These hubs should be further supported to by $\mathrm{MOH}$ to mitigate their persisting challenges of equipment breakdown and stock outs of reagents. Upgrading of some of these hub facilities would reduce the need to referral samples even further away from the requesting facilities and thereby reduce waiting times regionally. Quality improvement initiatives are still needed especially at hub, HC IV and hospital level laboratories so that they are able to perform tests according to standard.

Regarding availability of supplies, most of the laboratories, were well stocked with HIV testing kits, Malaria RDTs, TB reagents kits and gram stain kits. This was probably because of an additional source of funding both from donors and implementing partners in most of the laboratories. However, laboratories were less stocked with blood glucose test strips which are critical for detection of diabetes which is contributing to the increased non communicable diseases in Uganda. There were also less stock of chemicals, blood grouping anti sera, IgM tubex and glassware. It was further observed that stock outs of chemistry reagents were a critical challenge for the high-level laboratories. This was probably because government was the sole source of funding for most of the chemistry reagents. Similar observations were noted in Ethiopia where most of the laboratories did not have adequate chemistry reagents and chemicals to do all the necessary tests and thus health decisions could be possibly made based on incomplete laboratory test results (14). Therefore, there is need to ensure that high level laboratories are equipped with adequate vital and essential supplies especially for the advanced tests since these constitute the main referrals. Hub laboratories are officially recognized in the laboratory service delivery structure, however, there is no specified list/menus for essential and vital supplies for hubs. This makes these laboratories vulnerable to missing out on the most critical supplies and hence compromises their ability not be able to perform some tests. Therefore, there is need to develop and institute an essential supplies list of supplies menus for hub laboratories to ensure that the most vital and essential supplies are supplied to boost functionality.

\section{Conclusion}

In this study it was revealed that many laboratories performed well regarding the basic tests of malaria, HIV, urine and stool analysis but poorly with regard to availing advanced tests like chemistry, electrolytes and full blood counts in laboratories which are mandated to perform such tests including some of the hub laboratories and high-level laboratories. To mitigate wastage and improve the quality of services provided by laboratories harmonized efforts to be made to repair existing equipment, train staff where 
needed and to provide necessary supplies for functionality. The goal should be to make Hub laboratories fully functional while addressing supplies and capacity gaps where equipment exist at lower levels of care.

\section{List Of Abbreviations}

HC Health Center

HIV Human Immunodeficiency Virus

TB Tuberculosis

PCR Polymerase Chain Reaction

MoH Ministry of Health

CBC Complete blood Count

RRH Regional Referral Hospital

\section{Declarations}

\section{Ethics approval and consent to participate}

We obtained ethical approval from Makerere University School of Public Health Higher Degrees Research and Ethics committee (protocol number HDREC 364). All the participants consented before being interviewed.

\section{Consent for publication}

Not Applicable

\section{Availability of data and materials}

The datasets used and/or analyzed during the current study are available from the corresponding author on reasonable request.

\section{Competing interests}

The authors declare no competing interests

\section{Funding}

This study was funded by the Global fund to fight AIDS, tuberculosis and malaria.

\section{Authors' contributions}


SNK and ANK wrote the protocol of the study. NN and MA analyzed and interpreted the data. NN drafted the initial manuscript, all authors contributed to the first draft and all authors read and approved the final manuscript.

\section{Acknowledgements}

We would like to acknowledge the team of research assistants that helped in data collection and the staff at the Uganda Central Public Health Laboratories that gave input during the validation of the study findings.

\section{References}

1. Ndihokubwayo J-B, Maruta T, Ndlovu N, Moyo S, Yahaya AA, Coulibaly SO, et al. Implementation of the World Health Organization Regional Office for Africa stepwise laboratory quality improvement process towards accreditation. African journal of laboratory medicine. 2016;5(1):1-8.

2. HSSIP. The Health Sector Strategic and Investment Plan Uganda: Ministry of Health 2015.

3. Elbireer AM, Jackson JB, Sendagire H, Opio A, Bagenda D, Amukele TK. The good, the bad, and the unknown: quality of clinical laboratories in Kampala, Uganda. PLoS One. 2013;8(5).

4. Wertheim HF, Puthavathana P, Nghiem NM, van Doorn HR, Nguyen TV, Pham HV, et al. Laboratory capacity building in Asia for infectious disease research: experiences from the South East Asia Infectious Disease Clinical Research Network (SEAICRN). PLoS Medicine. 2010;7(4).

5. Nkengasong JN, Nsubuga P, Nwanyanwu O, Gershy-Damet G-M, Roscigno G, Bulterys M, et al. Laboratory systems and services are critical in global health: time to end the neglect? American journal of clinical pathology. 2010;134(3):368 - 73.

6. Amukele TK, Schroeder LF, Jackson JB, Elbireer A. Most clinical laboratory testing in kampala occurs in high-volume, high-quality laboratories or low-volume, low-quality laboratories: a tale of two cities. American journal of clinical pathology. 2015;143(1):50 - 6 .

7. Yao K, McKinney B, Murphy A, Rotz P, Wafula W, Sendagire H, et al. Improving quality management systems of laboratories in developing countries: An innovative training approach to accelerate laboratory accreditation. American journal of clinical pathology. 2010;134(3):401-9.

8. Elbireer AM, Opio AA, Brough RL, Jackson JB, Manabe YC. Strengthening public laboratory service in sub-Saharan Africa: Uganda case study. Laboratory Medicine. 2011;42(12):719 - 25.

9. Alemnji G, Zeh C, Yao K, Fonjungo P. Strengthening national health laboratories in sub-S aharan A frica: a decade of remarkable progress. Tropical Medicine \& International Health. 2014;19(4):450-8.

10. MOH. Standard Test Menu, Techniques and List of Supplies for Health Laboratories in Uganda Uganda: Ministry of Health 2017.

11. Reporter V. Government sets up 77 laboratory hubs. New Vision. 2015.

12. WHO. Laboratoryassessmenttool Geneva: World Health Organization 2012 [09-April-2020]. Available from: http://apps.who.int/iris/bitstream/10665/70874/3/WHO HSE GCR LYO 2012.2 eng.pdf?ua = 
1 \$ua $=1$.

13. Schroeder LF, Elbireer A, Jackson JB, Amukele TK. Laboratory diagnostics market in East Africa: a survey of test types, test availability, and test prices in Kampala, Uganda. PLoS One. 2015;10(7).

14. Getachew A, Cheneke W, Asres Y, Bekele S, Kebede E. Assessment of Coverage and Quality of Selected Clinical Chemistry Tests among Medical Laboratories of Health Facilities in Jimma Zone, South West Ethiopia. Journal of tropical medicine. 2019;2019.

15. Najjuka F, Opollo MS, Fred W-M. Uganda HIV Health Sector Review Ministry of Health, Government of Uganda, Kampala, Uganda: AIDS Control Program,, November 2010.; 2010. Available from: https://studylib.net/doc/7261438/health-sector-hiv-aids-review-2010.

\section{Figures}




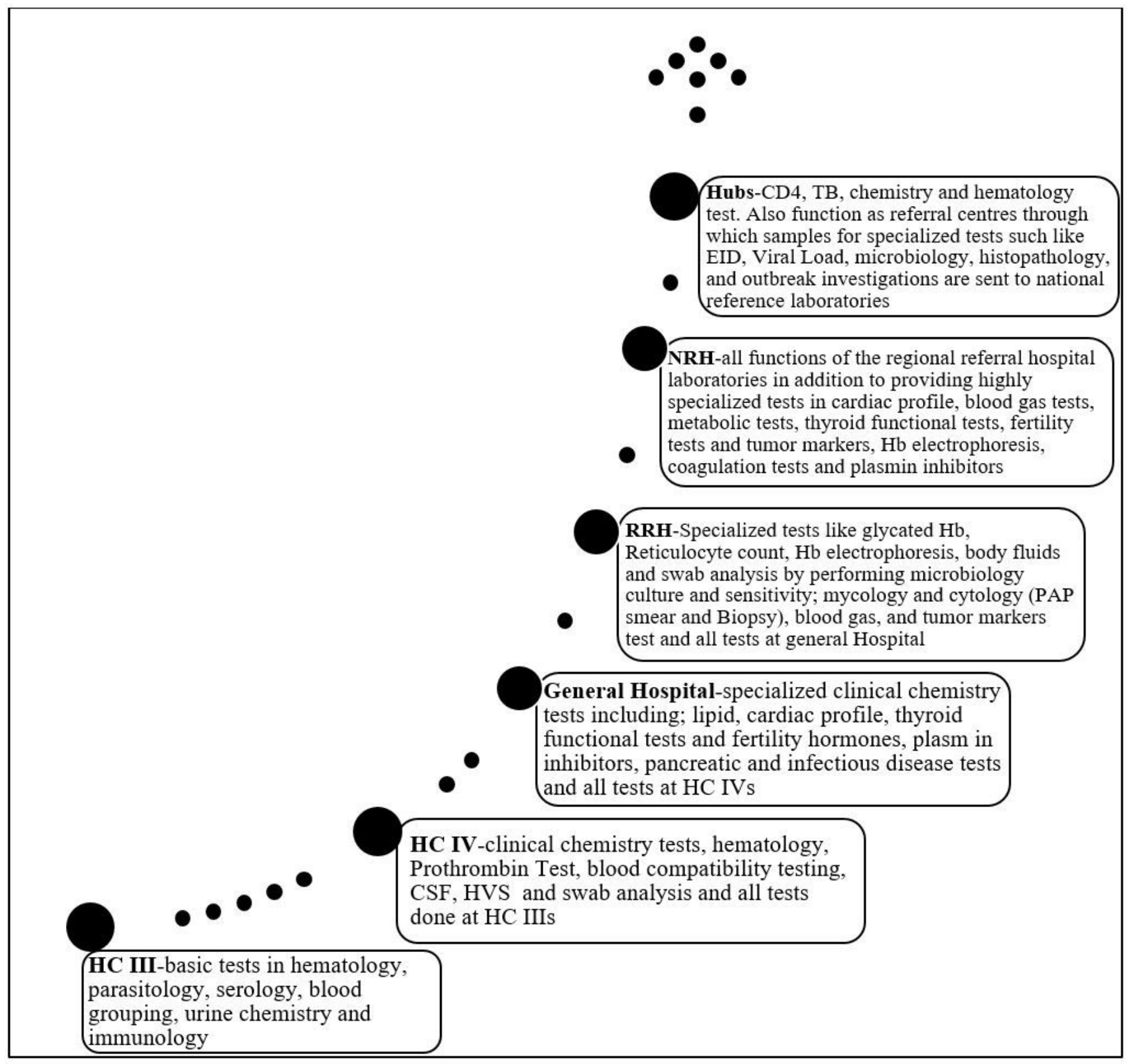

Figure 1

Laboratory test menus for Uganda (MOH, 2017) 